\title{
High precision transfer printing for hybrid integration of multi-material waveguide devices
}

\author{
John R. McPhillimy ${ }^{1}$, Benoit Guilhabert ${ }^{1}$, Charalambos Klitis ${ }^{2}$, Stuart May ${ }^{2}$, Martin D. \\ Dawson $^{1}$, Marc Sorel ${ }^{2}$, Michael J. Strain ${ }^{1}$ \\ ${ }^{1}$ Institute of Photonics, Dept. of Physics, University of Strathclyde, Glasgow G1 1RD, UK \\ ${ }^{2}$ School ofEngineering, University of Glasgow, Glasgow, Oakfield Avenue, Glasgow, G12 8LT, UK \\ Corresponding author email address: john.mcphillimy@strath.ac.uk
}

\begin{abstract}
We present a transfer printing technique with sub-100nm absolute placement accuracy. Hybrid integration of pre-processed membrane waveguide devices is achieved across a range of materials, including silicon, polymer and III-V devices.

OCIS codes: (130.0130) Integrated optics; (220.0220) Optical design and fabrication;
\end{abstract}

\section{Introduction}

Integrated photonics, across a range of material platforms has shown applications in fields from lab-on-a-chip sensing [1] to all-optical signal processing [2]. It is now becoming clear that to harness the full power of PIC "photonic integrated circuits" technology, multiple material platforms must be integrated into single chip-scale systems.

Transfer printing (TP) has been proposed as an attractive approach for hybrid integration with the ability to vertically assemble microscale devices onto virtually any material substrate in a massively parallel and scalable fashion. Recent demonstrations include III-V-on-silicon devices [3], nano-lasers [4], and $\mu$ LEDs [5]. However, TP lacks the nanometric absolute placement accuracy necessary for low-loss complex hybrid integration without utilising back-end processing [6]. We present a micro-assembly technique demonstrating integration of multiple material platforms with sub-100nm positional accuracy including vertically coupled polymer waveguides, silicon ( $\mathrm{Si}$ ) ring resonators, and both III-V nanowire lasers and $\mu$-disks integrated with waveguides.

\section{Nanoscale alignment accuracy}

The TP technique enables the micro-assembly of fully fabricated devices between different substrates in a highly controllable fashion (Fig 1a). A donor substrate contains suspended membranes with fully patterned waveguide structures (Fig 1b) as well as both substrates containing fabricated alignment markers with a non-centrosymmetric marker design, promoting sensitivity to variations in positional information. The absolute position of each alignment marker is calculated by cross-correlating to an identical virtual marker image of a known coordinate position within the TP system. By measuring the change in cross-correlation amplitude as a function of the TP system's translation stage, the absolute positions of donor and receiver substrates can be measured with high precision. Using this alignment method we can integrate individual photonic devicesvfrom different native substrates to sub-100nm positional accuracy (Fig 1c).

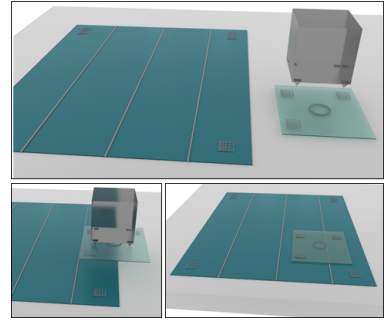

(a)

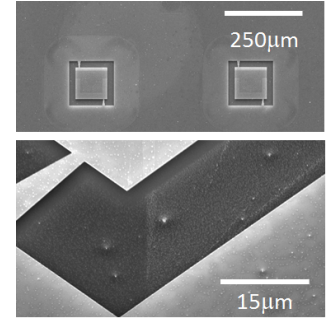

(b)

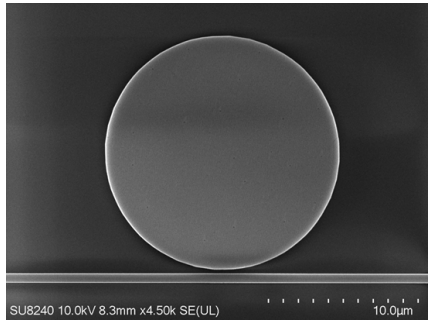

(c)

Fig. 1: (a) Schematic illustrating the micro assembly of multiple material substrates. (b) SEM images of Si suspended membranes situated on the donor substrate (c) SEM top-view image of AlGaAs micro-disk resonator transfer printed next to a SOI waveguide. 


\section{Micro-assembled photonic devices}

The micro-assembly of sub-micrometre waveguides across multiple material platforms is demonstrated. These include the vertical coupling of polymer waveguides through the bonding of thin-film glass substrates (Fig 2a) as well as the 3-dimensional integration of $\mathrm{Si}$ waveguides by the printing of pre-patterned suspended membranes (Fig 2b). Corresponding transmission spectra show the production of high quality ring resonator devices with low loss interfaces. The coupling strength between ring resonator and bus waveguide is controlled through the interlayer lateral alignment of the bonded waveguides.
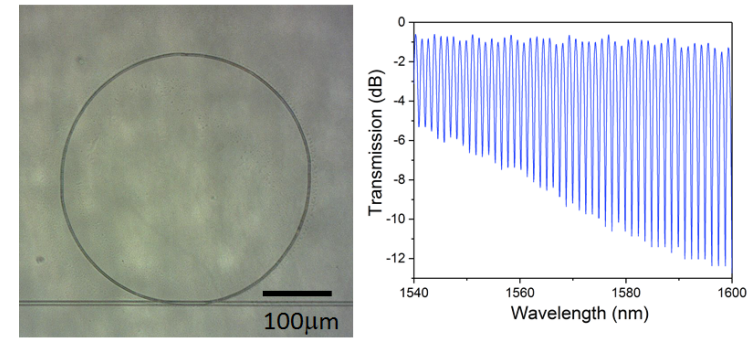

(a)
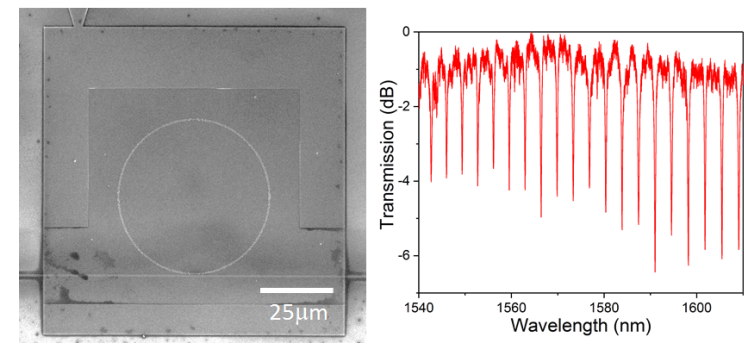

(b)

Fig. 2: (a) Vertically coupled polymer ring resonator and corresponding transmission spectrum. (b) Transfer printed membrane producing a vertically coupled Si ring resonator and corresponding transmission spectrum.

\section{Conclusions}

We present a nanoscale absolute alignment procedure which when used with a transfer printing technique enables the vertical micro-assembly of devices from a variety of material platforms. High quality optical devices are demonstrated.

\section{Acknowledgements}

This work was supported by the Engineering and Physical Sciences Research Council through Cornerstone (EP/L021129/1) and Parallel Heterogeneous Integration of III-V Devices on Silicon Photonic Chips (EP/P013570/1).

\section{References}

1. R. Wang, A. Vasiliev, M. Muneeb, A. Malik, S. Sprengel, G. Boehm, M.-C. Amann, I. Šimonyt, A. Vizbaras, K. Vizbaras, R. Baets, and G. Roelkens, "IIIV-on-Silicon Photonic Integrated Circuits for Spectroscopic Sensing in the $24 \mu \mathrm{m}$ Wavelength Range," Sensors 17, 1788 (2017).

2. G. Crosnier, D. Sanchez, S. Bouchoule, P. Monnier, G. Beaudoin, I. Sagnes, R. Raj, and F. Raineri, "Hybrid indium phosphide-on-silicon nanolaser diode," Nature Photonics 11, 297-300 (2017).

3. A. De Groote, P. Cardile, A. Z. Subramanian, A. M. Fecioru, C. Bower, D. Delbeke, R. Baets, and G. Roelkens, "Transfer-printing-based integration of single-mode waveguide-coupled III-V-on-silicon broadband light emitters," Optics Express 24, 13,754 (2016).

4. D. Jevtics, A. Hurtado, B. Guilhabert, J. McPhillimy, G. Cantarella, Q. Gao, H. H. Tan, C. Jagadish, M. J. Strain, and M. Dawson, "Integration of Semiconductor Nanowire Lasers with Polymeric Waveguide Devices on a Mechanically Flexible Substrate," Nano Letters 17, 5990-5994 (2017).

5. C. A. Bower, M. A. A. Meitl, B. Raymond, E. Radauscher, R. Cok, S. Bonafede, D. Gomez, T. Moore, C. Prevatte, B. Fisher, R. Rotzoll, G. A. Melnik, A. Fecioru, and A. J. Trindade, "Emissive displays with transferprinted assemblies of $8 \mu \mathrm{m} 15 \mu \mathrm{m}$ inorganic light-emitting diodes," Photonics Research 5, 23-29 (2017).

6. J. Yoon, S. M. Lee, D. Kang, M. A. Meitl, C. A. Bower, and J. A. Rogers, "Heterogeneously Integrated Optoelectronic Devices Enabled by Micro-Transfer Printing," Advanced Optical Materials 3, 1313-1335 (2015). 